\title{
Elementos del modelo educativo de la Universidad Tecnológica Fidel Velázquez utilizados en el proceso de enseñanza aprendizaje
}

\section{Elements of the educational model of the Fidel Velázquez Technological University used in the teaching-learning process}

\author{
HIDALGO-BAEZA, María del Carmen†**, HERNÁNDEZ-RAMÍREZ, María Guadalupe, MARTÍNEZ- \\ ROMÁN, Alejandro y CASAS-GÓMEZ, Víctor Manuel
}

Universidad Tecnológica Fidel Velázquez, Calle Emiliano Zapata S/N Col. El Tráfico Nicolás Romero Estado de México.

ID $1^{\text {er }}$ Autor: María del Carmen, Hidalgo-Baeza / ORC ID: 0000-0002-9768-3965, Researcher ID Thomson: S-7675-2018

ID $1^{\text {er }}$ Coautor: María Guadalupe, Hernández-Ramírez / ORC ID: 0000-0002-7001-8105, Researcher ID Thomson: S6810-2018

ID $2^{\text {do }}$ Coautor: Alejandro, Martínez-Román / ORC ID: 0000-0001-8009-6353, Researcher ID Thomson: S-7699-2018

ID $3^{\text {er }}$ Coautor: Víctor Manuel, Casas-Gómez / ORC ID: 0000-0002-0195-8910, Researcher ID Thomson: S-7776-2018

DOI: $10.35429 / J U M .2020 .12 .4 .11 .19$

Recibido Julio 15, 2020; Aceptado Diciembre 30, 2020

\begin{abstract}
Resumen
El objetivo de este trabajo es presentar la investigación que se realizó con los estudiantes de las carreras de Técnico Superior Universitario (TSU) en Infraestructura de Redes Digitales y Desarrollo de software Multiplataforma, donde se identificaron los elementos del modelo educativo de la Universidad Tecnológica Fidel Velázquez (UTFV) que se están utilizando en el proceso de enseñanza aprendizaje virtual como estrategia de aprendizaje en la pandemia de COVID-19. La investigación que se aplicó fue cuantitativa, se utilizó como instrumento de recopilación de información un cuestionario en Google con preguntas de respuesta cerrada y la muestra que se tomó fue sobre los estudiantes inscritos de primero a cuarto cuatrimestre. Donde se identificó que un $100 \%$ de los alumnos considero que la estrategia de aprendizaje virtual en UTFV es adecuada y permite que las sesiones de retroalimentación se lleven por meet, además de que el docente emplea como método de enseñanza las presentaciones electrónicas y Vodcast en la plataforma Classroom y las clases llevan a cabo en 2 modalidades síncrona y asíncrona. El resultado obtenido es proponer la generación de una plataforma donde se suban los Vodcast como un recurso didáctico que fortalece el aprendizaje y las competencias del TSU.
\end{abstract}

Educación a distancia, Estrategias, Plataforma educativa

\begin{abstract}
The objective of this work is to present the research that was carried out with the students of the Higher University Technician (TSU) careers in Digital Network Infrastructure and Multiplatform Software Development, where the elements of the educational model of the Fidel Velázquez Technological University were identified (UTFV) that are being used in the virtual learning teaching process as a learning strategy in the COVID-19 pandemic. The research that was applied was quantitative, a questionnaire on Google with closed-ended questions was used as an information gathering instrument, and the sample that was taken was on students enrolled from first to fourth semester. Where it was identified that $100 \%$ of the students consider that the virtual learning strategy in UTFV is adequate and allows the feedback sessions to be carried out by meet, in addition to the fact that the teacher uses electronic presentations and vodcast as a teaching method in the classroom platform and classes are carried out in 2 synchronous and asynchronous modes. The result obtained is to propose the generation of a platform where Vodcasts are uploaded as a didactic resource that strengthens the learning and competencies of the TSU.
\end{abstract}

Distance education, Strategies, Educational platform

Citación: HIDALGO-BAEZA, María del Carmen, HERNÁNDEZ-RAMÍREZ, María Guadalupe, MARTÍNEZ-ROMÁN, Alejandro y CASAS-GÓMEZ, Víctor Manuel. Elementos del modelo educativo de la Universidad Tecnológica Fidel Velázquez utilizados en el proceso de enseñanza aprendizaje. Revista de Gestión Universitaria. 2020. 4-12:11-19.

\footnotetext{
* Correspondencia al autor (Correo electrónico: mcarmen.hidalgo@utfv.edu.mx)

$\uparrow$ Investigador contribuyendo como primer autor.
} 


\section{Introducción}

La educación a Distancia, también conocida como "virtual" es constituida como una herramienta importante para atender temas educativos durante la pandemia por COVID19 en los diferentes niveles educativos en México.

De manera particular la UTFV, asume nuevas demandas, las cuales exigen cambios en los modelos presenciales, particularmente en la cobertura virtual como una acción institucional.

Un entorno virtual de aprendizaje integra herramientas que ofrece Internet y adapta a los alumnos y docentes en un aula virtual de aprendizaje eficiente compuesto por planes y programas de estudio, docentes, alumnos, material didáctico dinámico e interactivo, espacio educativo donde se promueve el trabajo individual y grupo, dispositivos tecnológicos para facilitar la docencia y el aprendizaje.

En el presente trabajo se muestra que elementos han sido efectivos en el proceso de enseñanza aprendizaje en línea en la Universidad Tecnológica Fidel Velázquez en el periodo 2020-2 y 2020-3, considerando el desarrollo de las clases en la plataforma de Google For Education.

El recurso digital de audio y video (Vodcasting) es considerado el más adecuado para las clases en línea que si los estudiantes tienen dudas pueden descargarlo y reproducirlo siendo parte del aprendizaje asincrónico.

En la UTFV, las sesiones de Meet y Zoom son utilizadas como herramientas en el aprendizaje síncrono, donde el docente y los estudiantes interactúan con su cuenta institucional y se imparten los temas de las asignaturas.

Para ello se propone utilizar las normas netiquetas, que sean adaptables al comportamiento de la red internet tanto para los docentes como los estudiantes, fomentar la motivación y la capacitación en otras plataformas educativas como Team, Schoology y otras.
En la modalidad presencial, Classroom tiene varias ventajas como: tener evidencia de las actividades realizadas por los alumnos y docentes, revisión del progreso de los alumnos, mantener materiales organizados de las materias impartidas y la facilidad de descargar la aplicación en el celular.

\section{Problema}

Debido a la pandemia se tuvo que cambiar de un modelo presencial a un modelo virtual en el periodo 2020-2 y 2020-3.

Por lo que se plantea el objetivo de identificar los elementos del modelo educativo que se están utilizado en el proceso de enseñanza aprendizaje en las carreras de TSU en Desarrollo de Software Multiplataforma e Infraestructura de Redes Digitales, para valorar las estrategias que se implementaron como: comunicación y retroalimentación, estrategias de enseñanza, competencias de aprendizaje en los estudiantes y recursos de Internet.

La investigación se aplicó a 68 estudiantes pertenecientes a las carreras de TSU en Desarrollo de Software Multiplataforma e Infraestructura de Redes Digitales.

Los problemas encontrados respecto a la comunicación e impartición de clases en línea durante la pandemia se muestran en el gráfico 1. Comunicación e impartición de clases en línea y son los siguientes:

- El correo electrónico no se considera un medio útil de retroalimentación.

- $\quad$ Fallas en el servicio de Internet.

- La computara se comparte con todos los integrantes de la familia.

- $\quad$ Falta de recursos económicos.
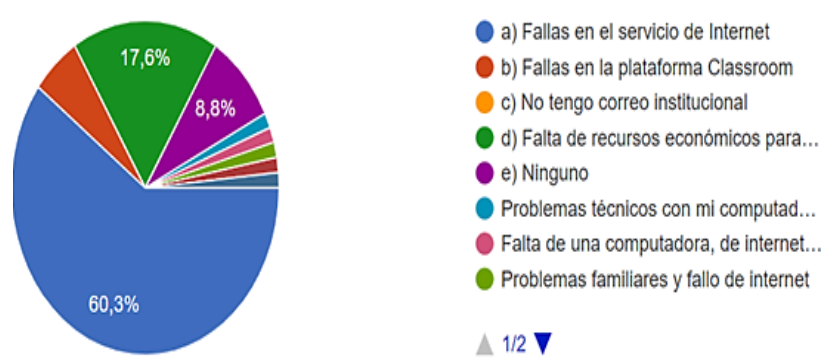

Gráfico 1 Comunicación e impartición de clases en línea 


\section{Objetivo}

Identificar los elementos del modelo educativo de la Universidad Tecnológica Fidel Velázquez utilizados en el proceso de enseñanza aprendizaje virtual.

\section{Objetivos Específicos}

- Identificar los medios de comunicación por el cual se recibe retroalimentación de un tema o tarea.

- Distinguir las diferentes estrategias de enseñanza que emplean los profesores.

- Conocer los recursos de Internet utilizados durante la pandemia de COVID-19.

- Mostrar las competencias que han generado los estudiantes con el aprendizaje en línea.

\section{Justificación}

El beneficio es que uno de los elementos que se está utilizando es Classroom de G Suite for Education, el cual es gratuito y se utiliza en la UTFV, donde los estudiantes y docentes no pagan por utilizarlo ya que cuentan con un correo institucional.

Otro beneficio es que la estrategia de enseñanza aprendizaje permite minimizar el riesgo de contagio de COVID-19 entre la comunidad universitaria.

Por lo tanto, la educación a distancia está permitiendo mayor uso de recursos de Internet y el autoaprendizaje en un ambiente colaborativo.

\section{Marco teórico}

La fundamentación teórica de la presente investigación se apoya en el concepto de educación, educación a distancia, estrategias de aprendizaje, recurso digital, reglas netiqueta.

\section{Concepto de educación}

La educación es tan antigua como el hombre, desde su aparición, el hombre se preocupó de criar y cuidar a sus hijos hasta que pudieran valerse por sí mismo, y es con este significado que surge el término educación.
Bello Díaz citado por Lorena Díaz (2012) define a la educación como "proporcionar al aprendiz la ayuda que le permita alcanzar niveles de desarrollo que él (o ella) no sea capaz de conseguir por sí mismo".

\section{Educación a Distancia}

Gabriel Valdez (s.f.) define a la educación a distancia como un sistema de enseñanza basado en el aprendizaje independiente y flexible, propiciado por un conjunto de medios y recursos didácticos que facilitan una comunicación bidireccional que sustituye la interacción personal en el aula del profesor y el alumno.

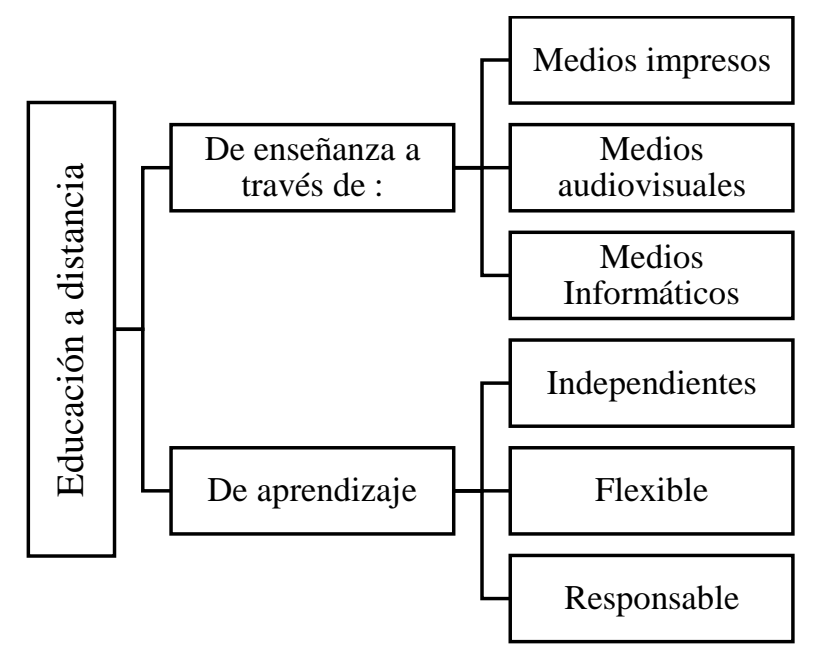

Figura 1 Concepto de educación a distancia Fuente: Elaboración propia

\section{Educación Virtual}

La educación virtual como la modalidad educativa que eleva la calidad de la enseñanza aprendizaje, y esto debido a que respeta su flexibilidad o disponibilidad, es decir, se puede canalizar para tiempos y espacios variables, logrando su mayor reconocimiento con la tecnología a través de métodos asincrónico y sincrónico y autoformación. (Lara 2002 citada, por Heedy y Martínez 2003)

Esta modalidad educativa surge durante el siglo XX, y tiene su auge en los años cincuenta y surge la Open University Británica y la Universidad Nacional a Distancia (UNED) española entre otras, sin embargo, es en la primera década del siglo XXI cuando crece de manera exponencial. 
En la figura 2, se identifican las características más sobresalientes de la educación a distancia.

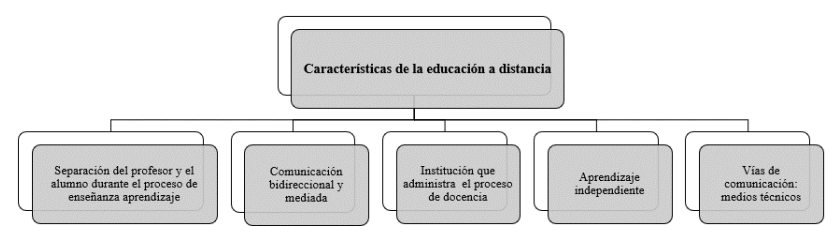

Figura 2 Elementos característicos de la educación a distancia

Fuente: Elaboración propia

En la enseñanza a distancia, la transmisión del conocimiento no requiere que el profesor y el alumno se encuentren cara a cara en un mismo lugar y al mismo tiempo.

La comunicación se produce a través de las distintas vías de comunicación: teléfono, correo electrónico entre otros, donde el profesor y el estudiante son a la vez emisores y receptores del mensaje educativo.

El uso de las tecnologías de la información y comunicación se ha venido incorporando, cada día más, al proceso de enseñanza como herramientas que facilitan la comunicación y el aprendizaje a distancia como: El teléfono, video, radio, Internet, teleinformática.

Los estudiantes son capaces de identificar lo que están dispuestos a prender con el acceso a una gran variedad de recursos, fomentando de esta manera su independencia e intensificando los medios de comunicación e información que den la oportunidad de aprender lo que es importante para ellos y puedan contribuir en su desarrollo personal.

\section{Educación asíncrona}

La educación a distancia asincrónica utiliza la computadora y la tecnología de informática para trabajar remotamente desde cualquier lugar en cualquier tiempo, es decir que la instrucción se ofrece y los estudiantes acceden a ésta en momentos diferentes o en cualquier momento que le resulte cómodo, sin tener que estar en línea al mismo tiempo.

Para J.F. Longoria (s.f.) en la educación asíncrona el docente requiere de:
Una organización efectiva del contenido de la materia asíncrono, que permita al estudiante realizar su trabajo sin tropiezos.

Claridad en lo que se espera de los estudiantes.

Disponibilidad de tiempo para atender la clase a través de correo electrónico y/o sesiones de asesorías a través de mensajería instantánea.

\section{Educación sincrónica}

La educación sincrónica es la modalidad tradicional presencial en el cual el estudiante y el docente llevan un dialogo conjuntamente a una hora determinada, y pueden realizar preguntas y respuestas, obtener retroalimentación inmediata y establecer una comunicación en tiempo real.

\section{Plataforma Educativa virtual}

Las plataformas educativas virtuales, también llamadas Sistemas de Gestión de Aprendizaje (LMS, Learning Management System), son aplicaciones Web que integran un conjunto de herramientas para el proceso de enseñanza aprendizaje en línea, permitiendo una enseñanza no presencial (e-learning) y/o mixta (blendedlearning), donde se combina la enseñanza en línea con asesorías. (Estrada, Zaldívar y Peraza, 2013).

Dentro de estas plataformas se puede colocar todo tipo de materiales y realizar acciones como: foros, wikis, recibir tareas del estudiante, desarrollar test, promover debates, crear chats, entre otros.

Actualmente existen un gran número de plataformas educativas virtuales tanto comerciales con un costo, mientras que otras son de software libre (open source) como: Chamilo, Atutor, Canvas LMS, Coursera, Iseazy, Miaulas, Moodle. 


\begin{tabular}{|c|c|}
\hline $\begin{array}{c}\text { Plataforma } \\
\text { Educativa Virtual } \\
\text { de Gratuitas }\end{array}$ & Características \\
\hline Classroom & $\begin{array}{l}\text { Esta dentro de G Suite for Education, es } \\
\text { gratuita y la usan centros de formación, } \\
\text { usuarios particulares que posean cuentas } \\
\text { de Gmail u organizaciones sin ánimo de } \\
\text { lucro. } \\
\text { Se diferencian cuatro perfiles en } \\
\text { Classroom: profesores, alumnos. Tutores } \\
\text { y administradores cada uno con } \\
\text { diferentes permisos o tipos de funciones } \\
\text { como para profesores crear y gestionar } \\
\text { clases, tareas y notas y tutores recibir un } \\
\text { resumen del trabajo de los alumnos por } \\
\text { correo electrónico. } \\
\text { Se puede usar en la versión web, siendo } \\
\text { compatible con los principales } \\
\text { navegadores o mediante las aplicaciones } \\
\text { para Androide e IOS }\end{array}$ \\
\hline $\begin{array}{l}\text { Moodle (Modular } \\
\text { Object-Oriented } \\
\text { Dynamic Learning } \\
\text { Environment). }\end{array}$ & $\begin{array}{l}\text { Usos para la educación superior como: } \\
\text { exámenes en línea, aprendizaje activo } \\
\text { (foros, conferencias y wikis), tiene una } \\
\text { aplicación gratuita Moodle Mobile o } \\
\text { Moodle Desktop- } \\
\text { Es proporcionado gratuitamente como un } \\
\text { programa de código abierto. } \\
\text { Proporciona un conjunto de herramientas } \\
\text { para soportar tanto el aprendizaje mixto } \\
\text { como en línea en } 120 \text { idiomas. } \\
\text { https://moodle.org/ }\end{array}$ \\
\hline Chamilo & $\begin{array}{l}\text { Es gratuita con una interfaz sencilla que } \\
\text { permite wikis, espacios para trabajar en } \\
\text { grupo, foro, red social interna entre otros. } \\
\text { https://chamilo.org/es/chamilo/ }\end{array}$ \\
\hline Coursera & $\begin{array}{l}\text { Plataforma de learning para empresas, } \\
\text { gobierno y universidades ofrece servicios } \\
\text { gratuitos. } \\
\text { https://www.coursera.org }\end{array}$ \\
\hline Sakai & $\begin{array}{l}\text { ES una plataforma creada por la } \\
\text { colaboración de universidades con sede } \\
\text { en América que ponen en marcha el } \\
\text { Proyecto Sakai para presentar una } \\
\text { alternativa con código abierto a Moodle }\end{array}$ \\
\hline $\begin{array}{c}\text { Plataforma } \\
\text { Educativa Virtual } \\
\text { Comerciales }\end{array}$ & Características \\
\hline BlackboarLMS & $\begin{array}{l}\text { Fundada } 1997 \text { que usan múltiples } \\
\text { universidades aunque también ofrece } \\
\text { soluciones para educación primaria, } \\
\text { formaciones complementarias en } \\
\text { empresas, sector público. } \\
\text { https://www.blackboard.com/ }\end{array}$ \\
\hline Microsoft Teams & $\begin{array}{l}\text { Controla tareas de clase, chats y } \\
\text { reuniones, pizarra digital, debates entre } \\
\text { otros. } \\
\text { Comparte y trabaja en equipo con } \\
\text { aplicaciones de Office } 365 \text { como Word, } \\
\text { Excel y PowerPoint directamente en } \\
\text { Teams. } \\
\text { https://www.microsoft.com/es- } \\
\text { mx/microsoft-365/microsoft- } \\
\text { teams/education }\end{array}$ \\
\hline eDucativa & $\begin{array}{l}\text { Plataforma de e-learning para cursos y } \\
\text { gestionar material educativo a través de } \\
\text { internet, ideal para escuelas, } \\
\text { instituciones, empresas o consultoría que } \\
\text { deseen impartir cursos a distancia o } \\
\text { apoyar la capacitación presencial. } \\
\text { www.educativa.com }\end{array}$ \\
\hline NEO & $\begin{array}{l}\text { Es una plataforma diseñada para todo tipo } \\
\text { de público y profesionales. } \\
\text { https://www.neolms.com/ }\end{array}$ \\
\hline
\end{tabular}

Tabla 1 Plataformas Educativas

\section{Estrategia de aprendizaje}

Virginia González (2003) señala que las estrategias de aprendizaje son un conjunto interrelacionado de funciones $y$ recursos, capaces de generar esquemas de acción que hacen posible que el alumno se enfrente de una manera eficaz a situaciones generales y específicas de su aprendizaje; que le permiten incorporar y organizar selectivamente la nueva información para solucionar problemas diverso orden.

\section{Recurso digital}

Los recursos educativos digitales son materiales compuestos por medios digitales y producidos con el fin de facilitar el desarrollo de actividades para el aprendizaje. (eduteka, 2017).

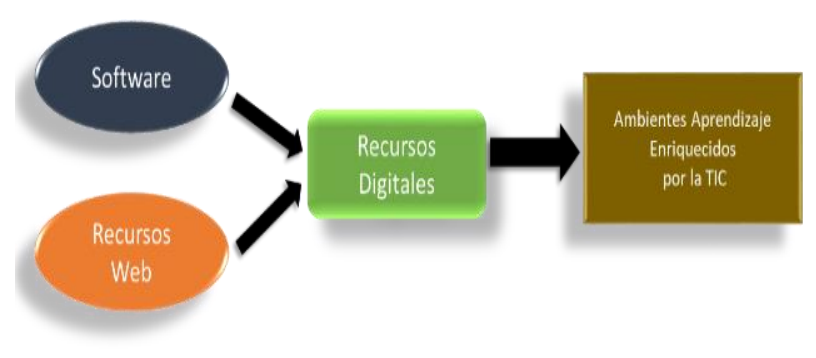

Figura 3 Recursos Digitales Fuente: Elaboración propia

Los recursos educativos ofrecen nuevas oportunidades como elementos que refuerzan el aprendizaje de los estudiantes.

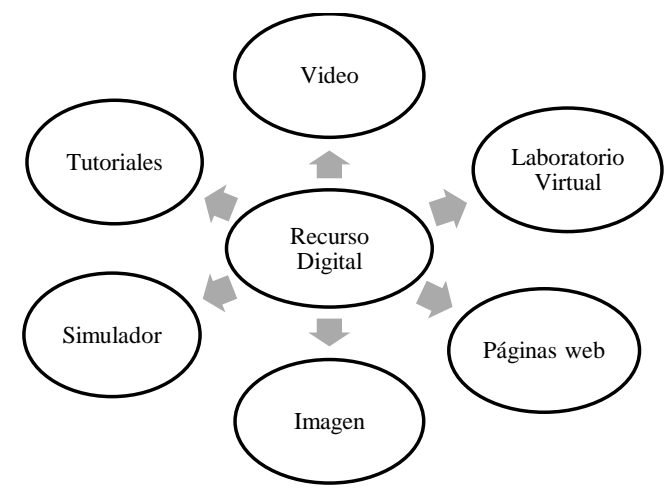

Figura 1 Recurso educativo digital

Fuente: Elaboración propia

\section{Vodcasting}

El vodcasting es un archivo de audio y video digital en formato MP4, que es distribuido mediante RSS que es descargado automáticamente o solicitándolo desde Internet para que el usuario lo pueda ver tanto en una computadora como en un reproductor portátil.

HIDALGO-BAEZA, María del Carmen, HERNÁNDEZ-RAMÍREZ, María Guadalupe, MARTÍNEZ-ROMÁN, Alejandro y CASASGÓMEZ, Víctor Manuel. Elementos del modelo educativo de la Universidad Tecnológica Fidel Velázquez utilizados en el proceso de enseñanza aprendizaje. Revista de Gestión Universitaria. 2020 
Se puede utilizar para: Documentales, guías descriptivas de sitios de interés, de contenido didáctico, explicito, de producción propia como actividad de clase, divulgación científica, congresos posibilidad de trasmitir ponencias, mesas redondas, programas de un canal de TV entre otros.

Solano (2010) define al Vodcast educativo como un medio didáctico con contenido educativo y que es creado a partir de un proceso de planificación didáctica.

\section{Netiqueta}

Netiquette es una palabra del francés etiquette y del inglés net (red) o network, castellanizado como netiqueta.

La Netiqueta es un conjunto de normas de comportamiento de los usuarios para comunicarse en Internet adaptada del mundo real a las tecnologías y al ambiente virtual.

El 28 de octubre de 1995 se elaboró un documento llamado RFC1855 (Request for Comments 1855) donde se definen las reglas de netiquetas de la red.

Virginia She, autora del libro NETiquette (1994), redactó una serie de normas de conducta en la Red, que se mencionan a continuación:

Regla 1: Nunca olvide que la persona que el mensaje es otro ser humano con sentimientos que pueden ser lastimados.

- Regla 2: Adhiérase a los mismos estándares de comportamiento en línea que usted sigue en la vida real.

Regla 3: Escribir todo en mayúsculas se considera como gritar y, además, dificulta la lectura.

Regla 4: Respete el tiempo y el ancho de banda de otras personas.

Regla 5: Muestre el lado bueno de sí mismo mientras se mantenga en línea.

Regla 6: Comparta sus conocimientos con la comunidad.

Regla 7: Ayude a mantener los debates en un ambiente sano y educativo.
- $\quad$ Regla 8: Respete la privacidad de terceras personas.

Regla 9: No abuse de su poder o de las ventajas que puede usted tener.

- Regla 10: Excuse los errores de otros, Comprenda los errores de los demás igual que usted espera que los demás comprendan los suyos.

\section{Metodología de investigación}

Mostrar los resultados de la encuesta aplicando el enfoque cuantitativo por categoría, con la finalidad de Identificar:

- $\quad$ Los recursos de Internet y las estrategias de aprendizaje empleadas para las clases virtuales.

Las competencias que han generado a través del aprendizaje en línea.

- Identificar la mejorar estrategia de comunicación y retroalimentación en los temas de las asignaturas.

El estudio se llevó a cabo con un tamaño de la población de 68 estudiantes de primero a cuarto cuatrimestre de las carreras de TSU en Desarrollo de Software Multiplataforma e Infraestructura de Redes Digitales.

Para la investigación se aplicó una encuesta y como instrumento se utilizó un cuestionario en Google drive, con 15 preguntas cerradas y se clasificaron las preguntas en categorías.

\section{Categoría competencia de los estudiantes}

En esta categoría el $33.8 \%$ de los estudiantes han desarrollado las siguientes competencias: autodisciplina, autoaprendizaje, análisis crítico y reflexivo, trabajo colaborativo y habilidad en el uso de las redes sociales. Ver gráfico 2.

La competencia de análisis crítico y reflexivo es de las más importantes y es la menor con un $4.4 \%$, por lo que es necesario buscar actividades que la refuercen, por ejemplo: promover las preguntas en la clase virtual (pregunta detonante), solución de problemas de su entorno social, debates entre otros.

HIDALGO-BAEZA, María del Carmen, HERNÁNDEZ-RAMÍREZ, María Guadalupe, MARTÍNEZ-ROMÁN, Alejandro y CASASGÓMEZ, Víctor Manuel. Elementos del modelo educativo de la Universidad Tecnológica Fidel Velázquez utilizados en el proceso de enseñanza aprendizaje. Revista de Gestión Universitaria. 2020 

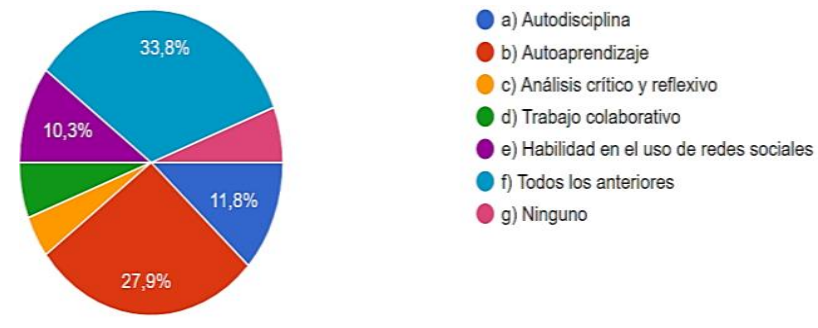

Gráfico 2 Competencias generadas

\section{Categoría recursos de Internet}

Se realizaron tres preguntas, la primera consistió esencialmente en los recursos de Internet que ocupan los estudiantes para complementar su aprendizaje, por ejemplo, el $41.2 \%$ prefiere consultar tutoriales $\mathrm{y}$ en las plataformas educativas virtuales el $98.5 \%$ Classroom. La impartición de las clases en línea se lleva a cabo síncrona y asíncrona en un $47.1 \%$ y el $7.4 \%$ lo hace de forma asíncrona mediante correo electrónico, chat o foro, ver gráfico 3.

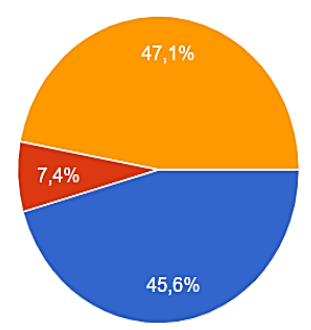

a) De manera sincrona utilizando videoconferencias

b) De manera asíncrona utilizando correo electrónico, chat o foro

c) Ambos a y b.

d) Ninguno

Gráfico 3 Impartición de clases en línea

\section{Categoría estrategias del profesor}

Esta categoría es una de las más importantes ya que las estrategias de enseñanza aprendizaje empleadas por los profesores van a impactar en los estilos de aprendizaje visual, auditivo y kinestésico, lo que puede influir en la comprensión de los temas.

Para la categoría se generaron 5 preguntas, la primera se refiere a las estrategias de enseñanza y estás fueron: simuladores, presentaciones electrónicas, práctica guiada y video de la sesión. Las presentaciones electrónicas es la estrategia de enseñanza que más utilizaron los docentes para sus clases con un $73.5 \%$ y la que menos es utilizada son los simuladores con $44.1 \%$. Ver grafico 4 .

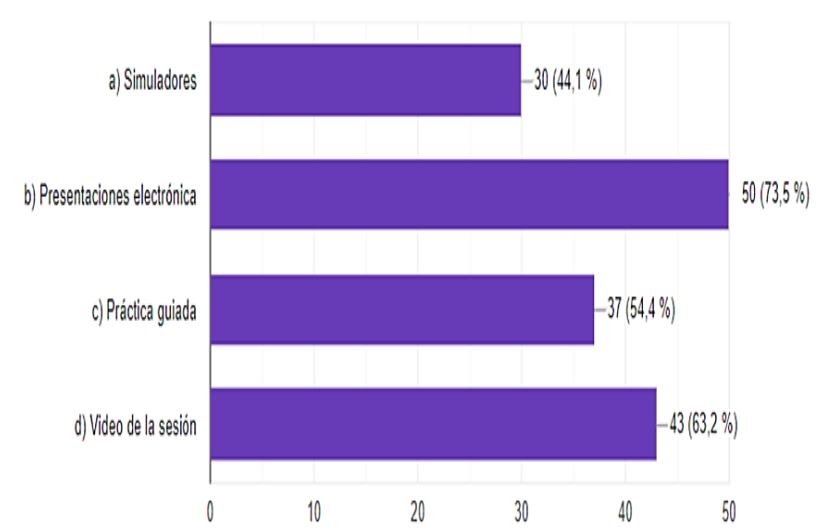

Gráfico 4 Estrategias del profesor

\section{Estrategia de enseñanza aprendizaje para una mejor compresión de temas}

En un $27.9 \%$, se determina que los videos realizados por el docente (vodcast) es la herramienta de autoaprendizaje que más ayuda a los estudiantes por su disponibilidad de fácil descarga, se pueden guardar en cualquier dispositivo de almacenamiento y es útil para los estudiantes que no asisten a la clase por algún motivo o quieren repasar la clase. Ver gráfico 5.
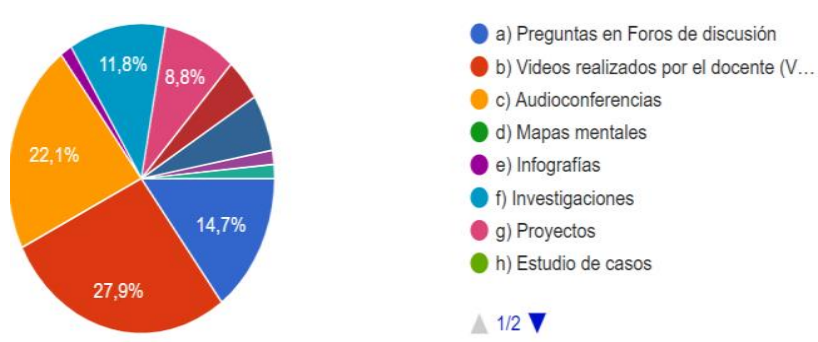

Gráfico 5 Estrategia de enseñanza para mejorar la compresión de temas

\section{Categoría comunicación y retroalimentación}

Para comunicación y retroalimentación se elaboraron 6 preguntas 3 están relacionadas con comunicación y las otras con retroalimentación. De acuerdo con las gráficas de comunicación el $76.5 \%$ de los estudiantes reciben retroalimentación sobre los temas y tareas asignadas por sus profesores mediante una videollamada en meet o Zoom y es el más adecuado. También consideran que el medio menos útil para recibir retroalimentación es mediante correo electrónico. Ver gráfico 6. 


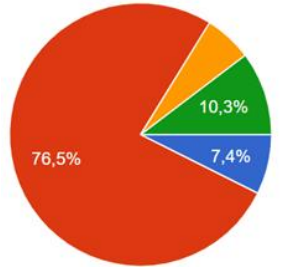

a) Mediante correo electrónico b) Mediante una videollamada (Google c) Por medio de

de un mensaje privado d) Video previamente grabado por el

Gráfico 6 Comunicación y retroalimentación

Con respecto a la retroalimentación de clases y tareas que reciben los estudiantes por parte de los maestros, el $88.2 \%$ respondió que si existe una retroalimentación por los distintos medios y consideran que es suficiente con 2 hrs. Es importante mencionar que cuando un alumno aclara sus dudas a tiempo, hay una mejor comprensión de los temas posteriores.

Ver gráfico 7.
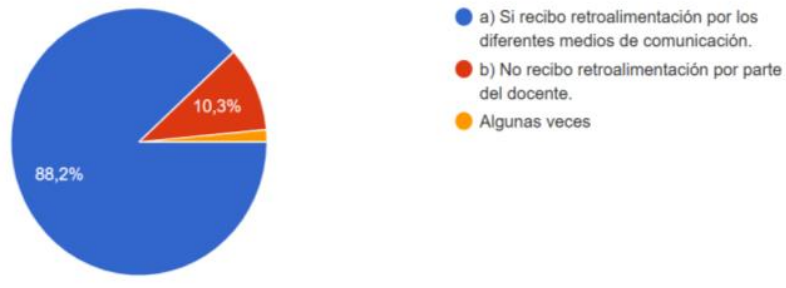

Gráfico 7 Retroalimentación por los docentes

\section{Conclusiones}

En este trabajo hasta el momento se presentó los elementos del modelo educativo de la UTFV, que se utilizado en el proceso de enseñanza aprendizaje virtual debido a la pandemia de COVID - 19.

En la investigación se obtuvo que el $92.6 \%$ de los estudiantes consideran que las estrategias implementadas por la Universidad Tecnológica Fidel Velázquez se evaluaron como buenas, ya que los alumnos(as) tienen una cuenta institucional y las clases se imparten en plataformas con varias herramientas como es el caso de Classroom y Microsot Teams.

Se cumple con el propósito de minimizar el riesgo de contagio y cumplir con la continuidad de sus estudios.

El 67.6\%, considera que su aprendizaje a distancia es bueno porque están dispuestos a aprender y pueden adaptarse a la situación que se está viviendo actualmente en el mundo.

\section{Referencias}

Díaz castro, L. (2012). Educación Virtual . Tlalnepantla, México, México.

Díaz Lara, G. (07 de 04 de 2016). Memorias de Producción de Multimedia Educativo. Obtenido de Memorias de Producción de Multimedia Educativo:

https://producciondemultimediaeducativocezy.b logspot.com/2016/04/el-vodcast-como-recursoeducativo.html

eduteka. (30 de abril de 2017). VII COngreso Virtual Iberoamericano de Calidad en Educación Virtual y Adistancia. Obtenido de VII COngreso Virtual Iberoamericano de Calidad en Educación Virtual y Adistancia: http://eduteka.icesi.edu.co/gp/upload/Recursos_ Educativos_Digitales_que_aportan_al_proceso _de_ensenanza_y_aprendizaje.pdf

Estrada Lizárrga, R., Zaldívar Colado, A., \& Peraza Garzón, J. F. (13). Análisis Comparativo de las Plataformas Educativas Virtuales Moodle y Dokeos. Revista Iberoamericana para la Investigación y el Desarrollo Educativo, 14.

Heedy, C., \& Martínez Uribe. (2003). La educación a distancia: sus características y necesidad en la educación actual. Educación, 21.

intef, I. N. (29 de 03 de 2017). educalab.es. Recuperado el 17 de 05 de 2019, de educalab.es: http://educalab.es/intef/tecnologia/recursosdigitales

Longoria, J. (s.f.). www.researchgate.net. Recuperado el 2020, de https://www.researchgate.net/pubwww.research gate.netlication/228607914_La_Educacion_en_ linea_El_uso_de_la_tecnologia_de_informatica _y_comunicacion_en_el_proceso_de_ensenanz a-aprendizaje

López García, J. C. (08 de 05 de 14). Universidad ICESI eduteka. Obtenido de Universidad ICESI eduteka: https://eduteka.icesi.edu.co/modulos/8/255/661/ 1 ?url=8/255/661/1

Rabajoli, G. (2012). Recurso digitales para el aprendizaje: una estrategia para la innovación educativa en tiempos de cambio. En G. Rabajoli, Digital (pág. 13). Montevideo Uruguay: Webinar 2012 - IPPE - UNESCO - FLACSO.

HIDALGO-BAEZA, María del Carmen, HERNÁNDEZ-RAMÍREZ, María Guadalupe, MARTÍNEZ-ROMÁN, Alejandro y CASASGÓMEZ, Víctor Manuel. Elementos del modelo educativo de la Universidad Tecnológica Fidel Velázquez utilizados en el proceso de enseñanza aprendizaje. Revista de Gestión Universitaria. 2020 
Valdez, G. (s.f.). www.academia.edu.

Recuperado el 2020, de

https://www.academia.edu/36552131/LA_EDU

CACI\%C3\%93N_A_DISTANCIA_CONCEPT

OS_Y_CARACTER\%C3\%8DSTICAS_UNID

AD_I 